\title{
Percepção da inovação sob a ótica do resource-based view
}

\section{RESUMO}

Luis Fernando Moreira Ifmoreira@ucs.br

Universidade de Caxias do Sul (UCS), Caxias do Sul, Rio Grande do Sul, Brasil

\section{Carlos Eduardo Schlindwein}

eduschilindwein@hotmail.com Sul, Rio Grande do Sul, Brasil.

\section{Daniel Faturi Silva}

Dfsilva20@ucs.br

Universidade de Caxias do Sul (UCS), Caxias do Sul, Rio Grande do Sul, Brasil.

\begin{abstract}
Buscar a inovação é algo considerado necessário no meio empresarial para alavancar os resultados organizacionais, pois a inovação procede da influência mútua de vários recursos de atitudes da organização. O objetivo deste artigo estudo é mensurar a percepção da inovação usando a teoria baseada em recursos através de uma escala gráfica segundo a visão baseada em recursos, por meio de um estudo quantitativo onde pode-se comprovar que os recursos tangíveis de tecnologia, financeiro e organizacional tem uma forte relação para inovação, a relação das variáveis analisadas e a visualização do gráfico de dispersão pode-se considerar correlação positiva forte. A contribuição de cunho social também foi relevante para a presente pesquisa, onde pessoas do gênero feminino ganham menos que pessoas do gênero masculino.
\end{abstract}

PALAVRAS-CHAVE: Inovação, Visão baseada em recursos, Mensurar. 


\section{INTRODUÇÃO}

Bencke (2016) cita que a inovação tem papel fundamental no crescimento e desenvolvimento de economias e sociedades. Pela natureza de seu caráter histórico e evolutivo, se apresenta como um tema complexo, com diferentes abordagens teóricas, paradigmáticas e modelos conceituais que apresentam definições e propõem métodos, formas e ferramentas consideradas como ideais para o seu desenvolvimento.

Segundo Crossan e Apaydin (2010), inovação é o processo de transformação de ideias em novidades como um produto novo ou melhorado, serviço ou processo para se destacar no mercado. Quandt, Bezerra e Ferraresi (2015) afirmam que a inovação resulta da interação de vários recursos e atitudes da organização. Para que se tenha êxito e promoção da competitividade, é fundamental a análise sobre o que a instituição oferece e onde está a lacuna na sociedade.

A visão baseada em recursos, alicerçada em estudos de autores como Penrose (1959), Wernerfelt (1984), Barney (1991), entre outros, tem como objetivo o estudo interno das variações da alocação de recursos e forças das instituições, entendendo que o alto desempenho é oriundo das rendas resultantes dos recursos específicos e dos lucros de posicionamento no mercado, Lin et al. (2012). Johnson, Scholes e Whittington (2007) citam que a visão baseada em recursos está ligada à exploração da capacidade estratégica de uma organização através de seus recursos e possibilidades, focados em trazer vantagem de mercado.

Araújo, Borges e Menezes (2018) afirmam que as empresas buscam a inovação como maneira de fomentar a sua produtividade, o seu desempenho e a sua competitividade no meio em que estão inseridas. Sendo assim, o objetivo do estudo é mensurar a percepção da inovação usando a teoria baseada em recursos através de uma escala gráfica. Para tal, o documento está dividido nas seguintes partes: além da presente seção apresentando a introdução, tem-se na sequência o referencial teórico apresentando os principais conceitos sobre os temas inovação e visão baseada em recursos, seguindo o artigo é apresentado a metodologia aplicada no estudo, após são expostos os resultados obtidos com a pesquisa e por último as considerações finais.

\section{REFERENCIAL TEÓRICO}

Na presente seção, serão apresentados os temas inovação e visão baseada em recursos, trazendo alguns conceitos sobre os mesmos definidos em estudos científicos durante o passar dos anos.

\section{INOVAÇÃO}

A discussão sobre a origem da inovação é pautada em duas vertentes, a primeira entendida por autores é de que a inovação foi "empurrada pela tecnologia", e a segunda possibilidade é tratada por pesquisadores como sendo a 
inovação "puxada pelo mercado", segundo Laim (2001). Rothwell (1994) traz a evolução da inovação dividindo-a em 5 fases, demonstradas na sequência.

Durante os anos de 1950 e meados de 1960, depois da segunda guerra mundial, a economia foi impulsionada pelas altas taxas de crescimento da indústria. As empresas investiam em pesquisa e desenvolvimento com foco em desenvolvimento de produtos e os colocavam no mercado para consumo. Esta fase foi marcada por ser empurrada pela tecnologia, conforme a figura 1.

Figura 1: Modelo de inovação (primeira geração)

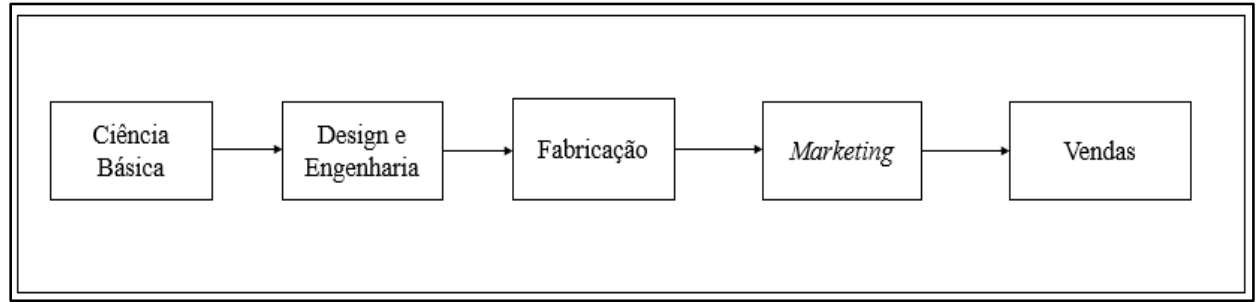

Fonte: Rothwell $(1994$, p. 8)

O segundo modelo de inovação surgiu nos meados dos anos de 1960 até início dos anos 70. Enquanto a produção industrial continuava a crescer, novos produtos continuavam a ser introduzidos no mercado, porém usavam tecnologias já existentes. Nesta fase os modelos de inovação começaram a observar a demanda que o mercado apresentava, a pesquisa era voltada a atender as necessidades apresentadas pelo mercado. Esta fase foi marcada pela inovação atendendo as necessidades do mercado, conforme figura 2 .

Figura 2: Modelo de inovação (segunda geração)

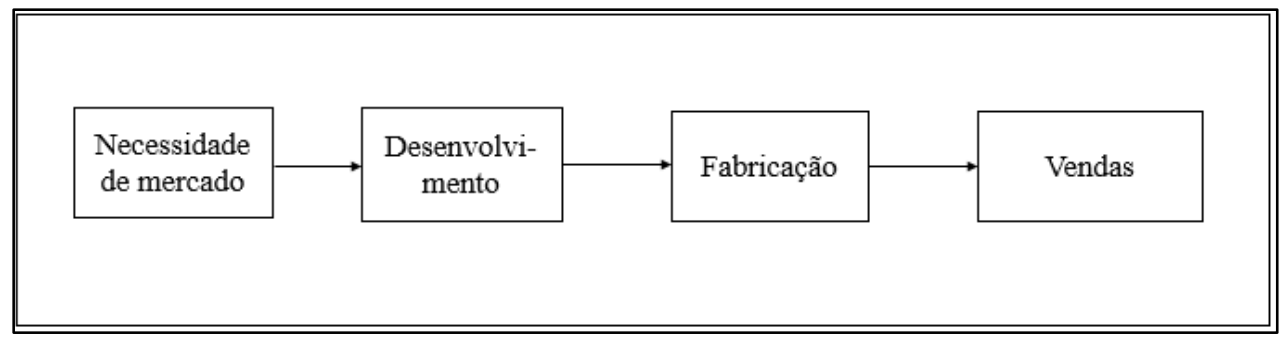

Fonte: Rothwell (1994, p. 9)

O terceiro modelo de inovação surge no início dos anos de 1970 e vai até a metade dos anos 80 . Com as crises de petróleo que afetaram os anos 70 , as empresas se viram obrigadas a racionalizar recursos e trabalhar com um controle maior de custos. Nesta fase que nasce o modelo de interação entre inovação com as necessidades de mercado e pesquisa e desenvolvimento, apresentado na figura 3. 
Figura 3: Modelo de inovação (terceira geração)

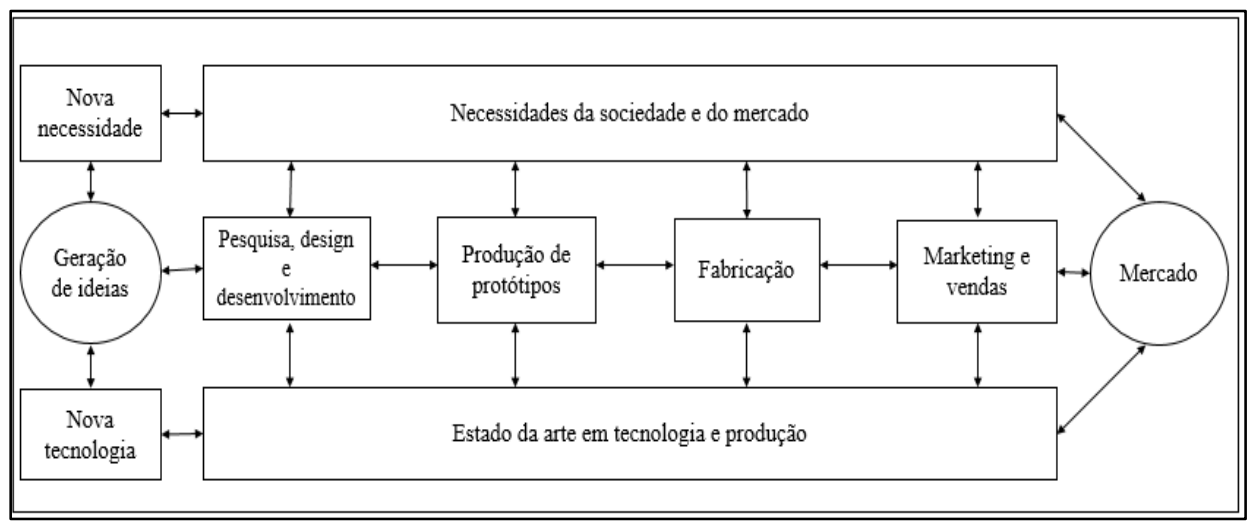

Fonte: Rothwell (1994, p. 10)

Rothwell (1994) diz que a quarta geração de inovação aconteceu nos anos de 1980 até início dos anos 90 . Período que foi marcado pela recuperação da economia, com produção de produtos com ciclo de uso menor e a indústria se adaptando a essa característica mercadológica. Período que foi marcado também pela exposição da indústria japonesa, destacando-se pela qualidade de produção.

A quinta geração se caracteriza por manter o que se construiu até então e com a preocupação da destruição do meio ambiente. Nesta fase a inovação junta pesquisa e desenvolvimento, trabalhando cada vez mais entre empresas do mesmo setor. A inovação acontece com a interação entre agentes externos e internos das empresas, como clientes, governo e fornecedores. A quinta geração é marcada por usar um conjunto de ferramentas com a finalidade de aumentar a eficiência dos processos e representam a inovação como um todo. A figura 4 representa a quinta geração de inovação.

Figura 4: Modelo de inovação (quinta geração)

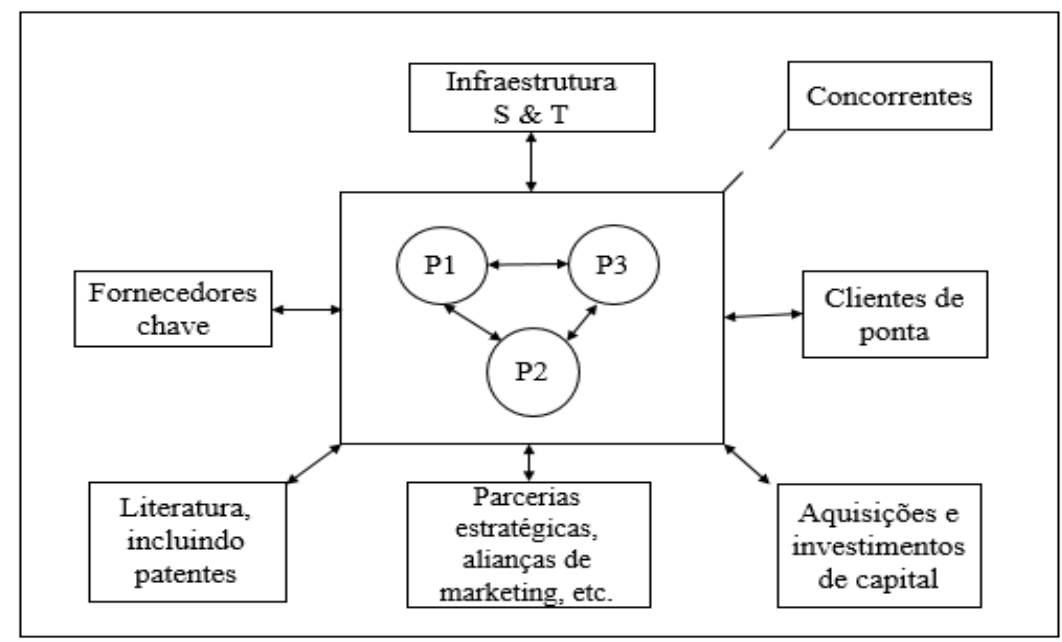

Fonte: Rothwell (1994, p. 27) 
Schumpeter (1934) começou a tratar a importância das inovações tecnológicas para que a economia de um país se desenvolva e atribuiu a elas, introduzidas pelos empreendedores inovadores, o papel principal para o início de um novo momento econômico. Sem o fator "inovação", a economia permaneceria parada, em um círculo fechado de bens e serviços e com crescimento estagnado.

Inovação segundo Schumpeter (1997), significa fazer as coisas de forma diferente no ambiente econômico. As inovações, segundo o autor, podem ser decorrentes de alguns motivos:

a) Introdução de um novo bem não conhecido pelas pessoas ou então, de uma nova qualidade de um bem já existente;

b) Abertura de um novo mercado, um mercado em que uma empresa nunca tivesse tido acesso antes, independente de este mercado já existir ou não;

c) Introdução de um novo método de produção, método que ainda não foi usado dentro do setor produtivo, mas que não necessita ser oriundo de uma descoberta científica;

d) Reorganização de uma indústria qualquer, como a criação ou a ruptura de um monopólio qualquer;

e) Descoberta de uma nova fonte de matéria prima ou de produtos semiacabados, independente desta fonte já existir anteriormente ou não.

Ao abordar inovação o OCDE (2005) diz que inovação é a implementação de um produto ou serviço novo ou significativamente aperfeiçoado, ou um processo, ou um novo método de marketing, ou um método organizacional novo nas práticas de negócios, na maneira como é organizado o local de trabalho ou nas relações externas. Segundo OCDE (2005), tem-se 4 dimensões de inovação, descritas a seguir:

a) Inovação de produto, é a introdução de um bem ou serviço novo ou aperfeiçoado no que concerne a suas características ou usos previstos. Incluemse melhorias significativas em características técnicas, componentes e materiais, softwares incorporados, facilidade de uso ou outras características funcionais.

b) Inovação de processo é a implementação de um modelo de produção ou distribuição novo ou aperfeiçoado. Incluem-se mudanças significativas em técnicas, equipamentos e/ou softwares.

c) Inovação de marketing é a implementação de um novo método de marketing com reais mudanças no desenvolvimento do produto ou em sua embalagem, no posicionamento do produto, em sua promoção ou na estipulação de preços.

d) Inovação organizacional é a implementação de um novo método organizacional nas práticas de negócios da organização, na organização do seu local de trabalho ou em suas relações externas.

Porter (2004) afirma que a inovação é pressuposta de mudanças na indústria. Segundo ele, a inovação pode acontecer em (a) inovação de produto, que apresenta alguma diferenciação em produto, podendo aparecer dentro da indústria ou fora dela; (b) processo, a forma de produção pode reduzir os custos 
do mesmo e alterar os custos fixos para produção; (c) marketing, possibilita buscar novos clientes e diminuir a sensibilidade a mudança de preços.

Nesse sentido, no presente trabalho, o objetivo é levantar os recursos considerados estratégicos para que a inovação aconteça de acordo com a teoria da Visão Baseada em Recurso. Recursos que levam as organizações a adquirir vantagens competitivas pelo conjunto de habilidades e rotinas essenciais e assim norteando para ser um gerador de inovação. O uso da teoria da RBV permitirá uma releitura criteriosa nos estudos organizacionais no meio empresarial, subsidiando dados e contribuições teóricas para futuras abordagens, bem como o entendimento de questões ainda pouco exploradas.

\section{VISÃO BASEADA EM RECURSO}

A teórica da RBV indica que os recursos internos da organização signifiquem nascentes de vantagens competitivas (BARNEY, 1986; 1991). A unidade fundamental de análise da RBV é composta pelos recursos e habilidades controladas pela firma, que contêm todos os predicados (sejam eles tangíveis ou intangíveis) que a certifiquem a definir e praticar estratégias (BARNEY e HESTERLY, 2004).

Um estudo de Salim, Rahman e Wahab (2019) através de uma revisão sistemática de literatura aborda as questões de recursos eficientes financeiros para gerar eco inovações. A RBV contribui para mensurar estes recursos nas organizações pelos seus serviços (BARNEY, 1986; 1991). Os recursos são importantes pelos serviços que podem prestar, e, em geral, um único recurso pode ser utilizado ou pode apresentar um número amplo de serviços, a depender da maneira como é empregado (PENROSE, 1959). Os recursos tangíveis são ativos, ou bens, que podem ser quantificados, enquanto que os intangíveis são aqueles relacionados à história da organização, tendo sido aglomerados no decorrer do tempo e de difícil repetição pela concorrência. (HITT et al., 2001)

Atos de competitividade de uma organização tendem a produzir resultados positivos sobre o seu papel no mercado, alocando-a em caráter de vantagem sobre suas concorrentes. Surge assim um ambiente de concorrência, por meio de uma série de atuações e respostas competitivas por parte das organizações que atuam num mesmo mercado. É anunciado que haja máxima rivalidade quando as empresas empregarem recursos mais semelhantes ou tiverem mercados em comum (HITT, IRELAND, HOSKISSON, 2008).

A visão baseada em recursos analisa a maneira pela qual os recursos organizacionais são cultivados e combinados e as razões que determinam a realização de uma vantagem sustentável na sua locação (CERTO e HODGE, 2007). Na RBV, a análise interior é seu objeto de empenho, os recursos são unidades de análise que consentem a compreensão, ao nível da organização, das vantagens competitivas. Nem todos os recursos da organização são essencialmente estratégicos; a condição estratégica é alcançada quando os recursos passam a ser carregadores de diferenciais qualitativos positivos em afinidade ao uso dos concorrentes (SALIM; RAHMAN e WAHAB, 2019; BLUME, 2008).

De acordo com Hitt, Ireland \& Hoskisson (2008) e Branco \& Rodrigues (2006) categorizam os recursos tangíveis em quatro grupos: 
(i). Financeiros: dizem respeito à capacidade de financiamento e de gerar receita. Um estudo empírico que aborda a inovação e recursos financeiros é o de Lee et al (2019), onde investigou-se a eficácia da inovação em sistemas financeiros na competência tecnológica do setor empresarial na Coreia. De acordo com Clark \& Allen (2012) quanto à melhoria das práticas financeiras, há um aumento gradual no valor das ações de empresas.

(ii). Organizacionais: referem-se à estrutura de planejamento, controle e coordenação. Segundo Searcy \& Elkhawas (2012) que o tema de seu estudo focou em ajudar as corporações a identificar áreas de melhorias futuras.

(iii). Tecnológicos: como patentes, marcas registradas, direitos autorais e segredos comerciais, que são formas de tangibilizar o conhecimento. $O$ estudo de Yu (2018), onde o desenvolvimento de uma plataforma SPOC (Small Private Online Course) baseada na ideia do MOOC (Massive Open Online Course), foi aplicada ao ensino misto de vários cursos em universidades locais da China. Foi explorado principais tecnologias de identificação no reconhecimento facial baseadas no cálculo de emoções, criação de cursos, edição e compartilhamento, análise do comportamento de aprendizagem on-line. Recurso tecnologia leva a eficácia operacional fazendo com que as empresas melhorarem a qualidade de seus produtos e serviços (GALLAUGHER, 2007).

Os recursos conferem à organização força para criar empecilhos de entradas imperativas à manutenção da competência de mercado. Essas barreiras advêm devido aos defeitos naturais no mercado de recursos (BARNEY, 1986). Exclusivamente por meio de nascentes de recursos estratégicos, as barreiras incidem a existir, seja por economias de escala, patentes, reputação da marca, etc., seja por recursos que atribuem à organização vantagens competitivas que novos entrantes não têm e podem obter apenas pausadamente e/ou por meio de altos custos (SALIM; RAHMAN e WAHAB, 2019; GRANT, 1991).

$\mathrm{Na}$ abordagem da visão baseada em recursos as corporações são avaliadas como heterogêneas e a diferença entre elas reside no seu potencial de vantagem competitiva (TUNER et al., 2007). De acordo com Vial et al. (2011) mediram as opções organizacionais no contexto da produção familiar (equitação de lazer), empregando as teorias de Economia dos Custos de Transação (ECT) e a RBV. Ao perpetrarem isso, as corporações tendem a entrar em mercados onde os prérequisitos de recursos retribuem às suas competências de recursos. Além disso, para abrir o potencial de uma corporação para alcançar uma vantagem competitiva sustentável, recursos e competências devem ter sinergia (SPECKBACHER; NEUMANN; HOFFMANN, 2015).

O valor de potencialidade de ganho de um recurso depende, muitas vezes, do ajuste entre eles e em quais configurações são implantados. Isso permite concluir que recursos preciosos, raros, podem continuar inativos, a menos que sejam postos em influência mútua com outros recursos, mesmo os mais comuns.

\section{METODOLOGIA}

O método utilizado teve abordagem quantitativa com a coleta de dados por meio de pesquisa survey, que envolve um questionário que está no apêndice " $A$ " utilizando o software Google formulários e por meio impresso. De acordo com 
Richardson (1989), este método caracteriza-se pelo emprego da quantificação, tanto nas modalidades de coleta de informações, quanto no tratamento, através de técnicas estatísticas, desde as mais simples, até as mais complexas.

As pesquisas descritivas, por sua vez, têm por objetivo descrever criteriosamente os fatos e fenômenos de determinada realidade, de forma a se obter informações a respeito daquilo que já se definiu como problema a ser investigado (TRIVIÑOS, 2008). Para a análise dos dados serão utilizados, tabelas cruzadas, teste de QUI quadrado, matriz de correlação entre os itens da survey, teste de confiabilidade da escala usada e ANOVA utilizando o software de análise de dados quantitativos SPSS $22^{\circledR}$ para organizar os dados.

\section{TÉCNICA AMOSTRAL}

A survey foi aplicada com 102 respondentes onde teve-se 40 questionários por meio eletrônico e 62 por meio físico apresentando 15 questões. O canal de difusão desta survey foram redes sociais, duas instituições de ensino superior e uma escola técnica profissionalizante da pesquisa durante os meses de outubro e novembro de 2018.

\section{AMOSTRAGEM SISTEMATICA}

De acordo com Martins (2002) a amostragem sistemática trata-se de uma variação da amostragem simples, que é conveniente quando a população está ordenada segundo algum critério. Como por exemplo, uma lista telefônica ou lista de presença escolar.

\section{DIMENCIONAMENTO AMOSTRAL}

Segundo Arango (2005), o dimensionamento amostral se menciona com a consignação do número de elementos mínimos indispensáveis de uma amostra a ser empregada em um estudo ou experiência de forma que este tenha legitimidade científica. $\mathrm{O}$ cálculo apresentado foi através da formula $\mathrm{n}=\mathrm{Z2} \times \mathrm{P} \times \mathrm{Q}$ $\times \mathrm{N} /$ e2 $\times(\mathrm{N}-1)+\mathrm{Z2} \times \mathrm{P} \times \mathrm{Q}$, que está representado no Quadro 1:

\begin{tabular}{|l|r|}
\hline 1). $Z$ = Nivel de Confiança & $95 \%$ \\
\hline 2). P = Quantidade de Acerto esperado (\%) & $90 \%$ \\
\hline 3). Q = Quantidade de Eno esperado (\%) & $5 \%$ \\
\hline 4). N = População Total & 135 \\
\hline 5). e = Nivel de Precisão (\%) & $3 \%$ \\
\hline Tamanho da amostra (n) $=$ & 102 \\
\hline
\end{tabular}

Fonte: Desenvolvido pelos autores (2020).

Quadro 2: Apresenta o escore Z do nível de confiança.

\begin{tabular}{|c|c|}
\hline Nivel de Confiança & Valor de $\mathbf{Z}$ \\
\hline $99 \%$ & 2,57 \\
\hline $95 \%$ & 1,96 \\
\hline $90 \%$ & 1,64 \\
\hline $80 \%$ & 1,28 \\
\hline
\end{tabular}

Fonte: Desenvolvido pelos autores (2020). 
O escore Z é o número de desvios-padrão pelo qual um valor disto a da média para mais ou para menos, representado no Quadro 2.

\section{ANÁLISE E DISCUSSÃO DOS RESUTADOS}

Os questionários recebidos foram compilados em uma base de dados eletrônica compatível com o software de análise SPSS 22. Inicialmente foram utilizadas tabelas cruzadas, teste de qui-quadrado, matriz de correlação Person entre os itens da survey, teste de confiabilidade da escala usada (alfa de Cronbach) e ANOVA com teste de cochram para verificar a significância dos dados apresentados neste estudo foi determinado um nível de significância onde o valor numérico que se refere ao valor de $\mathrm{p}$ correspondente à probabilidade de rejeitar $\mathrm{HO}$ quando é verdadeira. $\mathrm{O}$ grau de confiança é habitualmente escrito como $1-\alpha$, onde $\alpha$ é o complementar do grau de confiança, ou o nível de significância. Na compilação dos dados feito no software SPSS $22^{\circledR}$ temos um grau de confiança de 0.95 (ou 95\%) é o mesmo do que dizer que temos um nível de significância $\alpha=0.05$ é o mais utilizado em ciências sociais.

Níveis de significância, segundo D'Hainaut (1997, p.162):

1. $p>0.1$ estatística não significativa;

2. $p \leq 0.05$ estatística significativa;

3. $p \leq 0.001$ estatística muito significativa.

A confiabilidade de um instrumento de medida tem distintos aspectos. Existem, pois, distintas estatísticas para estimar confiabilidade, cada qual medindo um aspecto da conformidade do instrumento. Podem ser avaliadas:

i. A confiabilidade entre examinadores, ou seja, o grau com que diferente examinador vê em o mesmo fenômeno, usando o mesmo instrumento.

ii. A confiabilidade do teste-pré-teste, isto é, a relação das medidas feitas com o mesmo instrumento de medida, em períodos diferentes.

iii. A confiabilidade de forma paralela, que é a relação dos resultados de dois instrumentos diferentes, mas arquitetados da mesma maneira

$\mathrm{O}$ alfa de Cronbach ponderando as derivações anteriores e assumindo os mesmos pressupostos, mas sem limites no padrão de classificação dos itens, formaliza uma proposta de estimação de consistência interna a partir das variâncias dos itens e dos totais do teste por sujeito, que ficou conhecida como o índice "alfa" de Cronbach. A critério, como refere Cronbach e Shavelson (2004), a designação de "alfa" (inicialmente Alfa de Kuder-Richardson) pretendia apenas refletir a convicção do autor de que esta fórmula é puramente a primeira de um conjunto de cálculos necessários para medir as propriedades de uma escala para além da fiabilidade.

Segundo Murphy e Davidsholder (1988) a escala de fiabilidade é:

1.Fiabilidade inaceitável $<0,6$.

2.Fiabilidade baixa 0,7 .

3.Fiabilidade moderada a elevada 0,8-0,9. 
4.Fiabilidade Elevada $>0$.

O coeficiente alfa de Cronbach será útil para calcular a confiabilidade do teste por ter umas situações em que o pesquisador não tem a oportunidade de fazer outra entrevista com $\mathrm{o}$ indivíduo. $\mathrm{O}$ teste $\mathrm{Q}$ de Cochran na análise de delineamentos em blocos aleatorizados em que a variável de resposta pode admitir apenas dois valores possíveis, o teste $Q$ de Cochran é um teste estatístico não paramétrico para averiguar se tratamentos têm efeitos idênticos. (HAIR et al, 2002).

Primeiramente apresenta-se o cruzamento de tabelas, representado pela variável "Gênero" versus a variável "Renda" no quadro 3.

Quadro 3: Variável "Gênero" versus a variável "Renda"

\begin{tabular}{|c|c|c|c|c|c|c|c|c|c|}
\hline & \multicolumn{6}{|c|}{ Qual a sua renda? Salário mínimo é igual a RS: 954,00} & \multirow[b]{2}{*}{ Total } \\
\hline & & & $\begin{array}{l}1 \text { Salário } \\
\text { mínimo }\end{array}$ & $\begin{array}{l}\text { Acima de } \\
5 \text { salários }\end{array}$ & $\begin{array}{l}\text { Entre } 1 \text { e } 2 \\
\text { salários } \\
\text { mínimos }\end{array}$ & $\begin{array}{l}\text { Entre } 2 \text { e } 3 \\
\text { salários } \\
\text { mínimos }\end{array}$ & $\begin{array}{c}\text { Entre } 3 \mathrm{e} 4 \\
\text { salários } \\
\text { mínimos }\end{array}$ & $\begin{array}{c}\text { Entre } 4 \text { e } 5 \\
\text { salários } \\
\text { mínimos }\end{array}$ & \\
\hline \multirow{4}{*}{$\begin{array}{l}\text { 1. Qual } \\
\text { o seu } \\
\text { sexo? }\end{array}$} & \multirow[t]{2}{*}{ Feminino } & Contagem & $\overline{4}$ & 3 & 24 & 10 & 8 & 2 & 51 \\
\hline & & $\begin{array}{l}\text { Contagem } \\
\text { Esperada }\end{array}$ & 2,5 & 7,0 & 17,0 & 12,0 & 9,0 & 3,5 & 51,0 \\
\hline & \multirow[t]{2}{*}{ Masculino } & Contagem & 1 & 11 & 10 & 14 & 10 & 5 & 51 \\
\hline & & $\begin{array}{l}\text { Contagem } \\
\text { Esperada }\end{array}$ & 2,5 & 7,0 & 17,0 & 12,0 & 9,0 & 3,5 & 51,0 \\
\hline \multirow[t]{2}{*}{ Total } & & Contagem & 5 & 14 & 34 & 24 & 18 & 7 & 102 \\
\hline & & $\begin{array}{l}\text { Contagem } \\
\text { Esperada }\end{array}$ & 5,0 & 14,0 & 34,0 & 24,0 & 18,0 & 7,0 & 102,0 \\
\hline
\end{tabular}

Fonte: Software SPSS ${ }^{\circledast}$ (2020).

Análise feita pelos cruzamentos das variáveis acima, representou que vinte e quatro pessoas do sexo feminino ganham de 1 e 2 salários mínimos, duas pessoas do sexo feminino ganham entre 4 e 5 salários. Já em disparidades sete pessoas do sexo masculino ganham entre 4 a 5 salários mínimos, quatorze homens ganham entre 2 e 3 salários mínimos. Constata-se a diferença de salários entre os gêneros apontado pelo estudo de Cristina Bruschini (1999) consolidou análises sobre os ofícios das profissionais femininas que têm algum conceito na nossa sociedade, tais como engenharia, direito, arquitetura e medicina. $\mathrm{O}$ teste qui-quadrado de Person representa a significância da variável "Gênero" versus a variável "Renda".

Quadro 4: variável "Gênero" versus a variável "Renda"

\begin{tabular}{|l|r|r|rr|}
\hline & \multicolumn{1}{|c|}{ Valor } & \multicolumn{1}{c|}{ df } & Significância Sig. (2 lados) & \\
\hline Qui-quadrado de Pearson & $14,311^{1}$ & & 5 & \\
Razão de verossimilhança & 14,948 & 5 &, 011 \\
N de Casos Válidos & 102 & & & \\
\hline
\end{tabular}

Fonte: Software SPSS ${ }^{\circledR}$ (2020).

O teste apresentou o quanto categoria gêneros versus renda quão é provável a diferença analisada é adequada para as amostras não pareadas/emparelhadas onde apontou 0,014 que de acordo D’Hainaut (1997) é 
estatisticamente significativa $p \leq 0.05$. Em um segundo momento foi analisada a variável "Gênero" versus a variável "Escolaridade" representada no quadro 5.

Quadro 5 - variável "Gênero" versus a variável "Escolaridade"

\begin{tabular}{|c|c|c|c|c|c|c|c|c|c|c|}
\hline & \multicolumn{7}{|c|}{ 2. Nivel de Escolaridade? } & \multirow[b]{2}{*}{ Total } \\
\hline & & & $\begin{array}{l}\text { Médio } \\
\text { completo }\end{array}$ & $\begin{array}{c}\text { Médio } \\
\text { incompleto }\end{array}$ & $\begin{array}{c}\text { Pós } \\
\text { graduado } \\
\text { completo }\end{array}$ & $\begin{array}{c}\text { Pós } \\
\text { graduado } \\
\text { incompleto }\end{array}$ & $\begin{array}{c}\text { Pós } \\
\text { graduado } \\
\text { incompleto }\end{array}$ & $\begin{array}{l}\text { Superior } \\
\text { completo }\end{array}$ & $\begin{array}{c}\text { Superior } \\
\text { Incompleto }\end{array}$ & \\
\hline \multirow{4}{*}{$\begin{array}{l}1 \text { Qual } \\
\text { o seu } \\
\text { sexo? }\end{array}$} & Feminino & Contagem & 2 & 4 & 5 & 0 & 1 & 6 & 33 & 51 \\
\hline & & $\begin{array}{l}\text { Contagem } \\
\text { Esperada }\end{array}$ & 4,5 & 3,0 & 6,0 & 2,0 & 1,5 & 7,0 & 27,0 & 51,0 \\
\hline & Masculino & Contagem & 7 & 2 & 7 & 4 & 2 & 8 & 21 & 51 \\
\hline & & $\begin{array}{l}\text { Contagem } \\
\text { Esperada }\end{array}$ & 4,5 & 3,0 & 6,0 & 2,0 & 1,5 & 7,0 & 27,0 & 51,0 \\
\hline \multirow[t]{2}{*}{ Total } & & Contagem & 9 & 6 & 12 & 4 & 3 & 14 & 54 & 102 \\
\hline & & $\begin{array}{l}\text { Contagem } \\
\text { Esperada }\end{array}$ & 9,0 & 6,0 & 12,0 & 4,0 & 3,0 & 14,0 & 54,0 & 102,0 \\
\hline
\end{tabular}

Fonte: Software SPSS ${ }^{\circledR}$ (2020).

O teste evidenciou o quanto categoria gêneros versus escolaridade quão é provável a diferença analisada é adequada para as amostras não pareadas/emparelhadas onde apontou 0,086 que de acordo D'Hainaut (1997) é estatisticamente não significativa $p \leq 0.05$. Nesta análise ficou evidenciado que trinta e três pessoas do sexo feminino possuem graduação incompleta, seis graduações completa, cinco pós-graduação completa. Foi possível também ver a diferença de busca de qualificação entre pessoas de gêneros diferentes, conforme o estudo Cristina Bruschini (1999). Pessoas do sexo masculino apresentarão os seguintes dados: vinte e uma pessoas possuem superior incompleto, oito pessoas superior completo e sete pós-graduação completa denotando em valores mais expressivos que as pessoas do gênero feminino. $O$ teste qui-quadrado de Person representa a significância da variável "Gênero" versus a variável "Escolaridades" representado no quadro 6.

\begin{tabular}{|l|r|r|rr|}
\hline & \multicolumn{1}{|c|}{ Valor } & df & \multicolumn{2}{|c|}{ Significância Sig. (2 lados) } \\
\hline Qui-quadrado de Pearson & $11,063^{a}$ & 6 & &, 086 \\
Razão de verossimilhança & 12,817 & 6 & &, 046 \\
N de Casos Válidos & 102 & & & \\
\hline
\end{tabular}

Fonte: Software SPSS ${ }^{\circledR}$ (2020).

Em terceiro foi uma análise de matriz de correlação de Person entre os itens da amostra. Hair et al. (2006) sugerem que a amostra deve ser superior a 50 observações, sendo aconselhável no mínimo 100 casos para assegurar resultados mais robustos (HAIR et al, 2006). A razão entre o número de casos e a quantidade de variáveis deve exceder cinco para um ou mais (HAIR et al, 2006). Dancey e Reidy (2005) apontam para uma classificação ligeiramente diferente: $r=0,10$ até 0,30 (fraco); $r=0,40$ até 0,6 (moderado); $r=0,70$ até 1 (forte). 
Quadro 7: Matriz de correlação de Person do estudo.

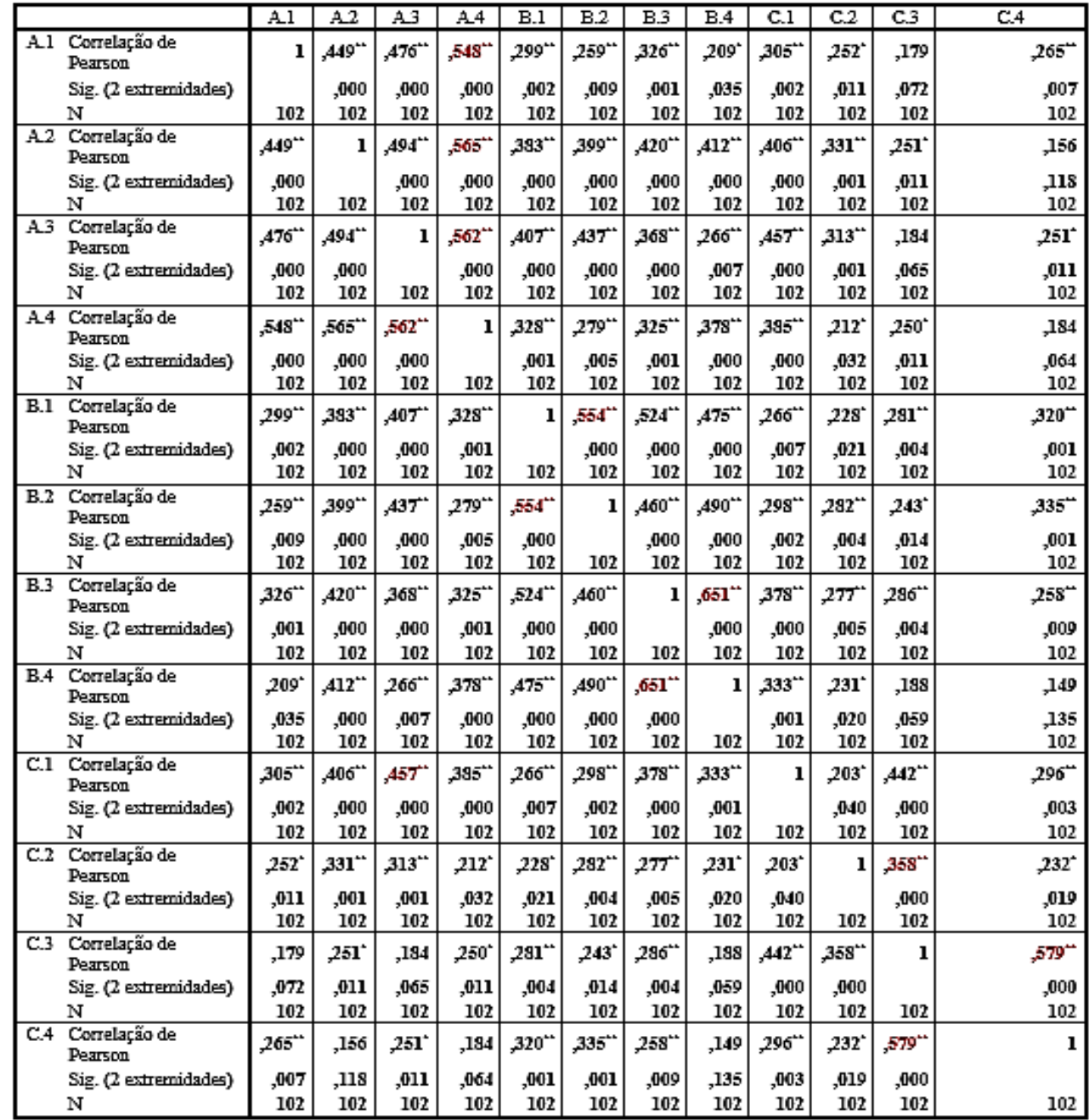

Fonte: Software SPSS ${ }^{\circledR}$ (2020).

Por quinto e último foi feito o teste ANOVA combinado com o teste de Cronbach para verificar a homogeneidade dos dados e compara a maior variância com as demais.

Quadro 6: Alfa de Cronbach da pesquisa

\begin{tabular}{|c|c|c|}
\hline $\begin{array}{c}\text { Alfa de } \\
\text { Cronbach }\end{array}$ & $\begin{array}{c}\text { Alfa de Cronbach com base em itens } \\
\text { padronizados }\end{array}$ & N de itens \\
\hline, 859 &, 862 & 12 \\
\hline
\end{tabular}

Fonte: Software SPSS ${ }^{\circledR}$ (2020).

Analise deliberada no quadro 7 deixa evidenciado a homogeneidade nos dados do estudo corroborando com o autor D'Hainaut (1997), $p \leq 0.05$ estatisticamente significativa e relevante. No quadro 7, ANOVA (ou Testes Robustos para a Igualdade de Médias), ficou em 0,001 mostrando homogeneidade dos dados. Valor for menor do que 0,05, as médias dos grupos são significativamente diferentes (HAIR et al, 2006). A partir do referencial teórico sobre inovação e visão baseada em recursos, apoiada no método quantitativo descritivo chegamos na representação gráfica apresentada na figura 5. 
Figura 5: Gráfico da dispersão das variáveis relacionadas.

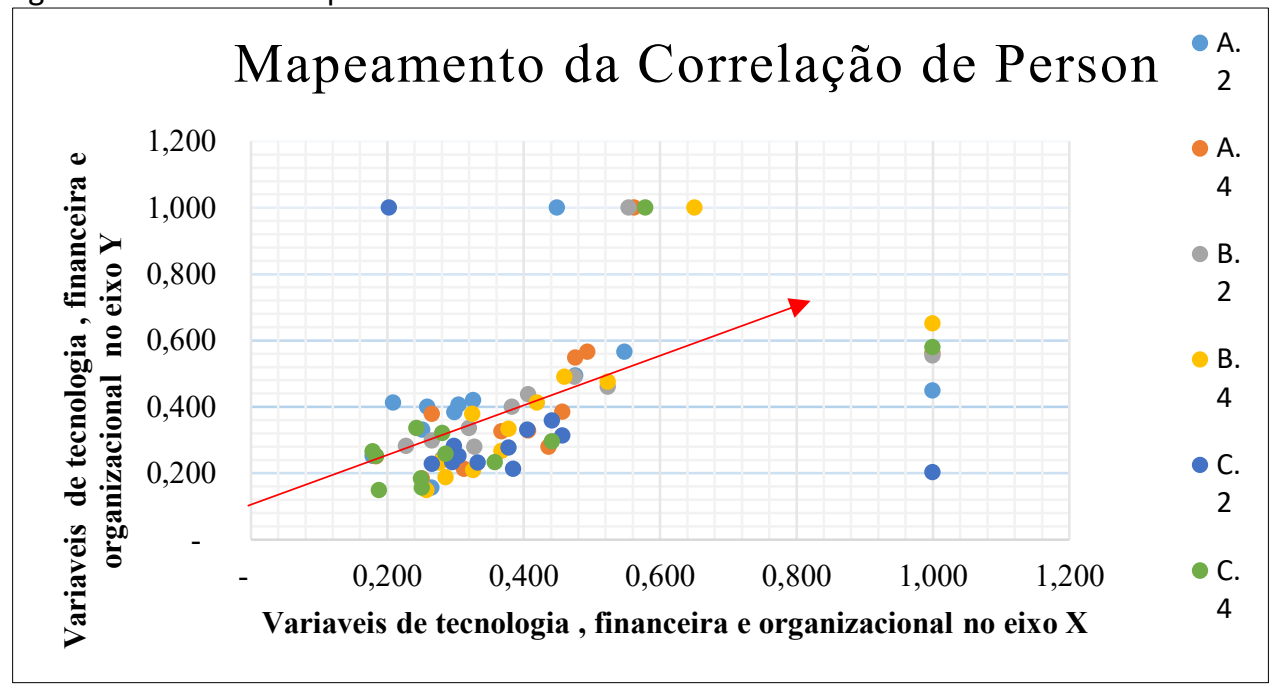

Fonte: Elaborada pelos autores (2020).

\section{CONSIDERAÇÕES FINAIS}

Estima-se que o coeficiente de correlação de Pearson e suas derivações são escolhidos em $95 \%$ dos casos para descrever o padrão de relacionamento entre variáveis ou para fazer inferências válidas para a população a partir de dados amostrais (CHEN e POPOVIC, 2002). O principal objetivo desse artigo é mensurar a percepção da inovação usando a teoria baseada em recursos através de uma escala gráfica. Procuramos apresentar as três dimensões da RBV: tecnológica, organizacional e financeira.

De acordo com a figura 5 pode-se visualizar a relação das variáveis analisadas e visualizar a sua dispersão que pode se considerar correlação positiva forte: há uma clara tendência nos dados. Quando a variável $X$ aumenta, é destinado conjuntamente um aumento na variável $Y$. O coeficiente de correlação de Pearson não diferencia entre variáveis independentes e variáveis dependentes. Dessa forma, o valor da correlação entre $X$ e $Y$ é o mesmo entre $Y$ e $X$. Schield (1995) aconselha que a correlação não se sobrepõe a distinção de causalidades simples ou recursiva. De acordo com a figura 5 pode-se avaliar de uma forma gráfica e visual o quadro 5 da correlação de Person e mensurar a percepção dos respondentes sobre a inovação e os recursos tangíveis aplicados $n$ este estudo onde foi atingido o objetivo principal.

A escala usada para medir a correlação já havia sido testada, o instrumento da survey foi adaptado de Koufteros (1999), bem como o alfa de Cronbach da pesquisa apresentado que ficou em ,0862 e de acordo com os autores Murphy e Davidsholder (1988) a confiabilidade é moderada a elevada. O artigo teve uma importância de cunho social, avaliando a área de formação de cada respondente e a renda, pode-se fazer uma análise de equiparação salarial entre pessoas de gêneros diferentes onde as pessoas do sexo feminino ganham menos do que o masculino baseado no estudo de Cristina Bruschini (1999).

A limitação do estudo foi o número reduzido de respondentes de 102 pessoas, ter abordado só três categorias abordados no instrumento de Koufteros 
(1999), onde ele aborda recursos tangíveis e intangíveis baseados em sete categorias: Categoria de recursos tangíveis: tecnológicos; Categoria de recursos tangíveis: físicos; Categoria de recursos tangíveis: organizacionais; Categoria de recursos tangíveis: financeiros; Categoria de recursos intangíveis: humanos; Categoria de recursos intangíveis: de inovação; Categoria de recursos intangíveis: de reputação. Para pesquisas futuras recomenda-se um número maior de respondentes, aplicação de todas as categorias do instrumento de Koufteros (1999), a pesquisa direcionada a um público alvo, mercados, organizações privadas e públicas. 


\title{
Perception of innovation from the resource- based view point of view
}

\begin{abstract}
Seeking innovation is something considered necessary in the business environment to leverage organizational results, since innovation comes from the mutual influence of various resources of the organization's attitudes. The objective of this study article is to measure the perception of innovation using the resource-based theory through a graphical scale according to the resource-based view, through a quantitative study where it can be proved that the tangible resources of technology, financial and organizational it has a strong relationship to innovation, the relationship of the variables analyzed and the visualization of the scatterplot can be considered a strong positive correlation. The social contribution was also relevant to the present research, where female people earn less than male people.
\end{abstract}

KEYWORDS: Innovation, Resource-based view, Measure. 
REFERÊNCIAS

ARANGO, H. G. Bioestatística - Teórica e Computacional, editora Guanabara Koogan, 2a edição, 2005, Rio de Janeiro/RJ.

ARAUJO, M. V.; BORGES, M. C.; MENEZES, D. C. As dimensões da inovação nas vinícolas orgânicas certificadas: uma abordagem a partir da visão baseada em recursos. 2018. crossref

BARNEY, J. B. (1986). Strategic factor markets: expectations, luck and business strategy. Management Science, 32(10), 1231-1241. DOI:

10.1287/mnsc.32.10.123. crossref

BARNEY, J. B. (1991). Firm resource and sustained competitive advantage. Journal of Management, 17(1), 99-120. DOI: 10.1177/01492063910170010. crossref

BENCK, F. F. EXPERIÊNCIA GAÚCHA DE PARQUES CIENTÍFICOS E TECNOLÓGICOS À LUZ DA TRÍPLICE HÉLICE. 2016. 351f. Tese (Doutorado em Administração) Universidade de Caxias do Sul, Brasil, 2016.

BARNEY, J. B., \& HESTERLY, W. (2004). Economia das organizações: entendendo a relação entre as organizações e a análise econômica. In S. R. Clegg, C. Hardy \& W. R.

BLUME, R. (2008). Explorando os recursos estratégicos do território para a vitivinicultura brasileira. Tese de Doutorado, Universidade Federal do Rio Grande do Sul, Porto Alegre, RS, Brasil.

BRANCO, M. C., \& RODRIGUES, L. L. Corporate social responsibility and resourcebased perspeztives. Journal of business Ethics. v, 69. n, 2. p, 111-113, 2006.

crossref

BRUSCHINI, CRISTINA LOMBARDI, MARIA ROSA. Médicas, arquitetas, advogadas e engenheiras mulheres em carreiras profissionais de prestígio. Revista Estudos Feministas. v. 7, n. 1-2, p. 9-24, 1999.

CERTO, S. TREVIS e HODGE, FRANK. Top Management Team Prestige and Organizational Legitimacy: An Examination of Investor Perceptions. Journal of Managerial Issues. Vol. 19, n. 4; p. 461-479, 2007. 
CRONBACH, L. J., \& SHAVELSON, R. J. My current thoughts on coefficient alpha and successor procedures. Educational and Psychological Measurement, v, 64. n, 3. P, 391-418, 2004. crossref

CHEN, PETER Y. \& POPOVIC, PAULA M. (2002), Correlation. London, Sage.

CLARK, T. S., \& ALLEN, D. S. Shareholder value from sustainability leadership: comparing valuation ratios whithin industry groups. International Research Journal of Finance and Economics, v. 89, p. 108-117,2012. crossref

CROSSAN, M. M.; APAYDIN, M. A Multi-Dimensional Framework of Organizational Innovation : A Systematic Review of the Literature. Journal of Management Studies, September, v. 47, n. 6, p. 1154-1191, 2010. crossref

DANCEY, CHRISTINE \& REIDY, JOHN. (2006), Estatística Sem Matemática para Psicologia: Usando SPSS para Windows. Porton Alegre, Artmed.

GRANT, R. The resource-based theory of competitive advantage: implications for strategy formulation. California Management Review, v, 33. n, 3. p, 114-135. DOI: $10.2307 / 41166664,1991$. crossref

GALLAUGHER, JOHN. Strategic Positioning and Resource-Based Thinking: Cutting Through the Haze of Punditry to Understand Factors Behind Sustainable, Successful Internet Businesses. International Journal of E-Business Research. v. 3. n, 3. p, 14-25, 2007. crossref

HAIR, JOSEPH F.; ANDERSON, ROLPH E.; TATHAM, RONALD L.; BLACK, WILLIAN C. Multivariate Data Analysis. 5a edição. Prentice Hall 2002

HAIR, Jr; BLACK, W. C; BABIN, B. J; ANDERSON, R. E e TATHAM, R. L. Multivariate Data Analysis. 6a edição. Upper Saddle River, NJ: Pearson Prentice Hall, 2006.

HITT, M. A., IRELAND, R. D., \& HOSKISSON, R. E. (2008). Administração estratégica. Tradução da 7ạ edição norte-americana. São Paulo: Cengane Learning

; IRELAND, R. D; HOSKISSON, R. E. Guest editors' introduction to the special issue: strategic entrepreneurship: entrepreneurial strategies for wealth creation. Strategic Management Journal, Vol. 21, 2001. p. 479-491. crossref

JOHNSON, G.; SCHOLES, K.; WHITTINGTON, R. Explorando a estratégia corporativa: texto e casos. Bookman Editora, 2007. 
KOUFTEROS, $X$. Testing a model of pull production: a paradigm for manufacturing research using structural equation modeling. Journal of Operations Management. v, 17, p.467-488, 1999. crossref

\section{LAIM, G. C. OS ATORES DE INOVAÇÃO NO QUÉBEC: UM ESTUDO}

EXPLORATÓRIO. 2001. 155f. Dissertação (Mestrado em Administração) Universidade de Caxias do Sul, Brasil, 2001.

$L E E$, J. et al. An empirical study on the effect of innovation financing on technology innovation competency: business performance of SMEs in Korea. Journal of Electronic Commerce in Organizations. v. 17, n. 1, p.1-15, 2019. DOI: 10.4018/JECO.2019010101. crossref

MARTINS, G. de A. Estatística Geral e Aplicada, editora Atlas, 2ª edição, 2002, p.157 - 200, São Paulo.

MURPHY, K. R., \& DAVIDSHOFER, C. O. (1988). Psychological testing: Principles and applications. Englewood Cliffs, New Jersey: Prentice Hall.

NORD (Eds.), Handbook de estudos organizacionais: ação e análise organizacionais (vol. 3). São Paulo: Atlas.

OCDE. Manual de Oslo: diretrizes para coleta e interpretação de dados sobre inovação. 3. ed. FINEP, 2005.

PENROSE, E. The theory of the growth of the firm. Oxford: Oxford University Press, 1959.

PORTER, M. Estratégia Competitiva: técnicas para análise de indústrias e da concorrência. 2. ed. Rio de Janeiro: Campus, 2004.

QUANDT, C. O.; BEZERRA, C. A.; FERRARESI, A. A. Dimensões da inovatividade organizacional e seu impacto no desempenho inovador: proposição e avaliação de um modelo. Gestão \& Produção, São Carlos, v. 22, n. 4, p. 873-886, 2015. crossref

RICHARDSON, R. J. Pesquisa social: métodos e técnicas. São Paulo: Atlas, 1989 
ROTHWELL, R. Towards the fifth-generation innovation process. International Marketing Reviw, v. 11, n. 1, p. 7-31, 1994. crossref

SALIM, N. A. RHAHMAN, M. N. WAHAB, ABD. A systematic literature review of internal capabilities for enhancing eco-innovation performance of manufacturing firms. Journal of Cleaner Production. v. 209, p. 1445-1460, 2019. crossref

SEARCY, C., \& ELKHAWAS, D. Corporate sustainability ratings: an investigation into how corporations use the Dow Jones Sustainability Index. Journal of Cleaner Production, v. 35, p. 79-92, 2012. crossref

SCHUMPETER, J. A. The theory of economic development. 1.ed. Harvard University Press, Cambridge, 1934

Teoria do desenvolvimento econômico: uma investigação sobre lucros, capital, crédito, juro e ciclo econômico. São Paulo: Nova Cultural, 1997.

SPECKBACHER, G.; NEUMANN, N.; HOFFMANN, W. H. Resource relatedness and the mode of entry into new businesses: internal resource accumulation vs. access by collaborative arrangement. Strategic Management Journal, v. 36, n. 11, p. 1675-1687, 2015. crossref

SCHIELD, MILO. (1995), "Correlation, Determination And Causality In Introductory Statistics". American Statistical Association, Section on Statistical Education. 1995.

TUNER, CRAIG A.; RYMAN, JOEL A. e CLARK, WILLIAN CLARK. The Anorexic Trend of Business: A Resource-Based View of Managerially Downsized Firms. Journal of Global Business Issues. Vol. 1, N. 2; p. 81-88, 2007.

TRIVIÑOS, A. N. da S. Introdução à pesquisa em ciências sociais: a pesquisa qualitativa em educação. São Paulo: Atlas, 2008.

YU,J. Research on Key Development Technologies for SPOC Platform. 2nd International Conference on Electronic Information Technology and Computer Engineering, EITCE 2018; Shanghai University. v. 232, p. 19, 2018. crossref

VIAL, C.; AUBERT, M.; PERRIER-CORNET, P. Les choix organisationnels des propriétaires de chevaux de loisir dans les espaces ruraux. Économie rurale. Agricultures, alimentations, territoires, n. 321, p. 42-57, 2011. crossref 
Recebido: 13 Mar. 2020

Aprovado: 14 Out. 2020

DOI: $10.3895 /$ gi.v16n4.11767

Como citar:

MOREIRA, L.F. et al. Percepção da inovação sob a ótica do resource-based view. R. Gest. Industr., Ponta Grossa, v. 16 ,

n. 4, p. 186-205, Out./Dez. 2020. Disponivel em: https://periodicos.utfpr.edu.br/revistagi

Correspondência:

Luis Fernando Moreira

Universidade de Caxias do Sul (UCS), Caxias do Sul, Rio Grande do Sul, Brasi

Direito autoral: Este artigo está licenciado sob os termos da Licença Creative Commons-Atribuição 4.0 Internacional.

\section{(c) (1)}

